\title{
Un autre regard sur les implications de l'étude DCCT
}

\author{
G. Krzentowski (1) ${ }^{(1)}$ L. Zhang ${ }^{(2)}$, A. Albert ${ }^{(2)}$, P.-J. Lefèbvre ${ }^{(3)}$ \\ (1) Service de Diabétologie et de Médecine Interne, CHU de Charleroi, Belgique. \\ (2) Département de Biostatistique, Université de Liège, Belgique. \\ (3) Département de Médecine, Service de Diabétologie, Nutrition et Maladies métaboliques, Université de Liège, Belgique.
}

Tirés à part : P.-J. Lefèbvre, Département de Médecine, Service de Diabétologie, Université de Liège, Belgique. e-mail : Pierre.Lefebvre@ulg.ac.be

\section{Another look at the implications of the DCCT study}

G. Krzentowski, L. Zhang, A. Albert, P.-J. Lefèbvre

Ann. Endocrinol., $2004 ; 65,5: 429-435$

The fundamental role of good metabolic control has been demonstrated in type 1 and type 2 diabetes. Nevertheless, clinicians often wonder why some patients under good metabolic control develop complications while others remain free of such complications, despite a poorly controlled disease.

The present study revisited material from the DCCT database, by classifying the 1441 patients as being under good or poor metabolic control if their $\mathrm{HbA}_{1 \mathrm{c}}$ mean level fell in the lower $\left(\mathrm{HbA}_{1} \leq 6.9 \%\right)$ or upper $\left(\mathrm{HbA}_{1} \geq 9.5 \%\right)$ quintile of the overall distribution of mean $\mathrm{HbA}_{1 c}$ levels observed in the DCCT population. The impact of metabolic control and of other potential factors related to the patient and his/her disease on the development and/or deterioration of complications, in particular diabetic retinopathy and nephropathy, was assessed. Although metabolic control is the major determinant of the risk of developing diabetic retinopathy and nephropathy, the study also emphasizes the significant role of other risk factors, in particularly $\mathrm{BMI}$, disease duration, micro-albuminuria, $\mathrm{HbA}_{1 c}$ at baseline, gender and age on such complications.

It is concluded that early control of the metabolic and clinical status of diabetic patients has major consequences on the evolution of the disease. Nomograms have been proposed to help the clinician in this task.

Key words: Good metabolic control, poor metabolic control, diabetic retinopathy, diabetic nephropathy.

\section{Un autre regard sur les implications de l'étude DCCT}

Le rôle fondamental du bon équilibre du diabète a été démontré tant en ce qui concerne le diabète de type 2 que celui de type 1. Néanmoins les cliniciens sont régulièrement confrontés à des patients qui, malgré un bon équilibre métabolique, développent des complications et d'autres qui, malgré un mauvais équilibre, n'en développent pas.

Nous avons donc repris les données de l'étude DCCT, avons réparti les 1441 patients de cette étude en quintiles en fonction de leur taux moyen d'HbA $\mathrm{A}_{1 c}$ durant l'étude, en considérant le quintile inférieur $\left(\mathrm{HbA}_{1 c} \leq 6,9 \%\right)$ comme " diabète bien équilibré » et le quintile supérieur $\left(\mathrm{HbA}_{1 \mathrm{c}} \geq 9,5 \%\right)$ comme " diabète mal équilibré ». Nous avons ensuite évalué l'impact non seulement de l'équilibre glycémique mais aussi d'autres facteurs caractéristiques du patient et de sa maladie afin de préciser s'ils exercent une influence sur l'apparition et/ou l'aggravation des complications, et plus particulièrement de la rétinopathie et de la néphropathie diabétiques. II ressort clairement de cette analyse post-hoc que l'effet de l'équilibre glycémique est fondamental mais que d'autres facteurs (Indice de Masse Corporelle, durée du diabète, micro-albuminurie, $\mathrm{HbA}_{1 c}$ au départ, âge et sexe) influent également significativement sur le devenir de ces redoutables complications.

Nous pouvons donc conclure qu'il est impérieux de diagnostiquer la maladie au plus tôt et de traiter au mieux les patients diabétiques non seulement en ce qui concerne leur équilibre glycémique mais aussi pour ce qui concerne les autres facteurs de risque. Des nomogrammes sont proposés pour aider le clinicien dans cette tâche.

Mots-clés : Bon contrôle métabolique, mauvais contrôle métabolique, rétinopathie diabétique, néphropathie diabétique.

\section{INTRODUCTION}

Le rôle fondamental du bon équilibre métabolique du diabète est reconnu de longue date en Europe, notamment suite aux résultats de l'étude de Pirart [10] publiée en 1978. Toutefois, l'étude DCCT [18], réalisée entre 1983 et 1989 et publiée en 1993, a eu un impact majeur et les consignes thérapeutiques actuelles dans le domaine du diabète de type $1[1,4]$ prennent cette étude pour référence.

L'expérience clinique confirme que le bon contrôle métabolique protège des complications micro- et macro-angiopathiques du diabète et que le mauvais contrôle en favorise l'apparition et le développement. Ces complications sont dévastatrices sur le plan humain et coûteuses sur le plan social puisqu'il est admis que la part la plus importante des coûts liés au diabète résulte directement de ces complications [2]. Cependant, les cliniciens reconnaissent aussi que les patients diabétiques ne sont pas égaux face au risque de complications, certains développant peu de complications alors que leur équilibre métabolique est mauvais tandis que d'autres en développent davantage malgré un bon équilibre de leur affection.

Face à ce constat paradoxal, nous avons réanalysé les données de l'étude DCCT rendues disponibles grâce au « National Technical Information Service (NTIS) » du « Department of Commerce » (USA). Le but de cette analyse était de vérifier 
scientifiquement la contradiction clinique évoquée cidessus et de rechercher d'éventuels facteurs de risque ou de protection vis-à-vis de la rétinopathie et/ou de la néphropathie diabétique. Le présent travail constitue une synthèse clinique des observations que nous avons publiées en détails par ailleurs $[26,27]$.

\section{LES DONNÉES DE L'ÉTUDE DCCT}

L'étude DCCT a recruté 1441 diabétiques de type 1 dans 29 centres cliniques entre 1983 et 1989. La durée moyenne de suivi ( \pm écart-type) était de 6,5 $\pm 1,6$ années. Tous les sujets diabétiques de type 1 âgés de 13 à 39 ans, avec une durée de diabète de 1 à 5 ans sans rétinopathie ni néphropathie à l'état basal, étaient considérés comme le groupe de prévention primaire; les sujets avec une durée de diabète de 1 à 15 ans avec rétinopathie et/ou néphropathie débutante étaient considérés comme le groupe d'intervention secondaire. Ces sujets ont été répartis de façon aléatoire dans un groupe de traitement « intensif » ou « conventionnel». Les détails des critères d'éligibilité et de randomisation ont été publiés par ailleurs [16, 17].

Dans les travaux que nous avons menés, le «bon contrôle métabolique » est défini comme le groupe des patients de l'étude DCCT présentant une valeur moyenne d'hémoglobine glycosylée $\left(\mathrm{HbA}_{1 c}\right)$ en cours d'étude $\leq 6,9 \%$, un seuil correspondant au percentile 20 de la distribution globale des taux moyens d'hémoglobine glycosylée des patients. De manière similaire, le "mauvais contrôle métabolique » est défini comme le groupe des patients de l'étude DCCT avec une valeur moyenne d'hémoglobine glycosylée en cours d'étude $\geq 9,5 \%$, ce qui correspond au percentile 80 de la distribution globale des taux moyens d'hémoglobine glycosylée. En d'autres termes, les patients avec un bon ou un mauvais contrôle métabolique sont ceux situés respectivement dans le quintile inférieur ou supérieur de la distribution des taux d'hémoglobines glycosylées de l'étude DCCT, indépendamment du type de traitement suivi, intensif ou conventionnel.

Dans notre étude sur la rétinopathie [26], la définition de cette complication correspondait à celle donnée dans l'étude originale [19]. Par ailleurs, comme seule était envisagée l'apparition ou la non apparition de cette complication en fonction du contrôle métabolique, il fut décidé de ne prendre en compte que les patients de la cohorte de " prévention primaire », c'est-à-dire ceux ne présentant pas de rétinopathie diabétique à l'entrée de l'étude. Parmi ceux-ci, 153 patients ont eu un «bon contrôle métabolique » et 166 un "mauvais contrôle métabolique ».
En ce qui concerne notre étude sur la néphropathie [27], cette complication fut définie comme l'existence d'une micro-albuminurie, c'est-à-dire une excrétion urinaire d'albumine (AER) $\geq 40 \mathrm{mg} / 24 \mathrm{~h}$, ce qui permit de prendre en compte non seulement les patients de la cohorte de prévention primaire mais aussi ceux de la cohorte d'intervention secondaire. Parmi ces patients, 277 figuraient dans le groupe « bon contrôle métabolique » et 268 dans le groupe "mauvais contrôle métabolique ».

Tant pour la rétinopathie que pour la néphropathie, les variables étudiées étaient: l'âge à l'entrée de l'étude, I'indice de masse corporelle (BMI), l'excrétion urinaire d'albumine (AER), l'apport calorique, la durée du diabète, le niveau d'éducation, le quotient intellectuel, le taux de $\mathrm{HbA}_{1 c}$ à l'entrée, le taux résiduel de Cpeptide, le profil moyen glycémique, l'apport nutritionnel en protéines, la dose totale d'insuline, les taux de cholestérol HDL et LDL, le taux des triglycérides, la pression artérielle moyenne, la durée de participation à I'étude, la glycémie moyenne, I'histoire familiale de diabète de type 1 , le statut marital, le tabagisme, le type de traitement (intensif ou conventionnel), le sexe et enfin la qualité du contrôle métabolique (bon ou mauvais) comme défini plus haut.

Les résultats ont été exprimés sous forme de moyenne \pm écart-type (SD) pour les variables quantitatives et en termes de proportions (\%) pour les variables catégorisées. Les analyses multivariées ont été réalisées par la méthode de régression logistique ou celle des risques proportionnels de Cox. Les résultats étaient considérés comme significatifs au niveau d'incertitude de $5 \%(P<0,05)$ après ajustement pour comparaisons multiples. Les calculs ont été réalisés à l'aide des logiciels statistiques SAS (version 8.2 pour Windows) et S-PLUS (version 6.2).

\section{UN AUTRE REGARD SUR LES DONNÉES DE L'ÉTUDE DCCT}

\section{Rétinopathie [26]}

Sur les 153 patients bien équilibrés $\left(\mathrm{HbA}_{1 \mathrm{c}} \leq 6,9 \%\right)$ durant l'étude DCCT, environ un patient sur dix $(9,8 \%)$ a développé une rétinopathie diabétique. Par contre, sur les 166 patients mal équilibrés $\left(\mathrm{HbA}_{1 \mathrm{C}} \geq 9,5 \%\right)$, près de la moitié $(42,8 \%)$ n'a pas développé de rétinopathie diabétique au terme de l'étude (tableau I). L'analyse statistique des données a montré qu'après avoir pris en compte le contrôle métabolique, seules trois variables permettaient d'expliquer ces pourcentages étonnants, à savoir le taux $\mathrm{d}^{\prime} \mathrm{HbA}_{1 \mathrm{c}}$ à l'entrée dans l'étude, le BMl et la durée de participation à l'étude (c'est-à-dire le reflet de l'exposition à un bon ou à un mauvais équilibre du diabète). 

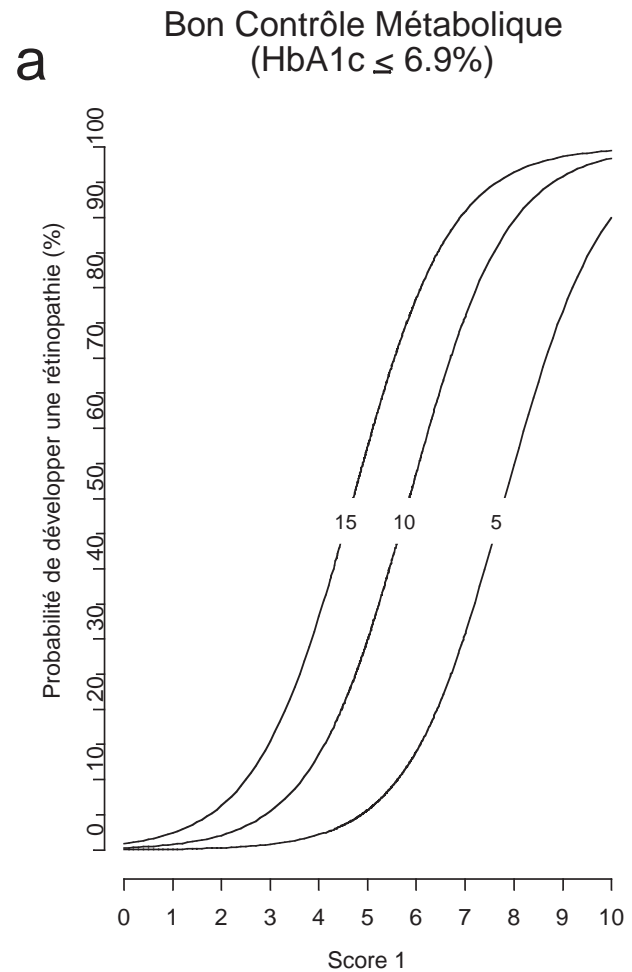

b Mauvais Contrôle Métabolique

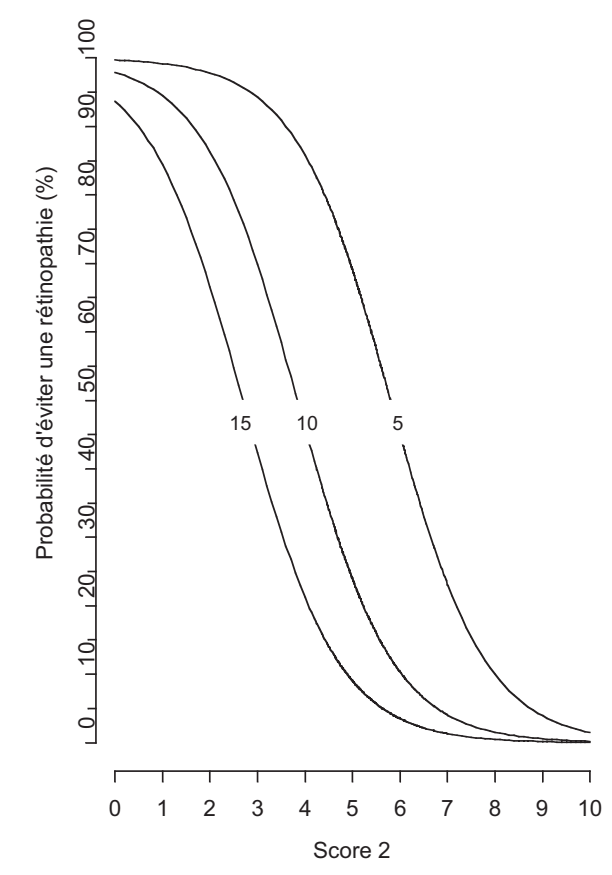

Figure 1 : a : Probabilité de développer à 5,10 ou 15 ans une rétinopathie diabétique sous bon contrôle métabolique $\left(\mathrm{HbA}_{1 c} \leq 6,9 \%\right)$ en fonction du score du patient; le score de risque $\left(S_{1}\right)$ est calculé à partir de l'équation: $S_{1}=0,314 \times \mathrm{HbA}_{1 c}+0,108 \times B M I$. b : Probabilité d'éviter à 5 , 10 ou 15 ans une rétinopathie diabétique sous mauvais contrôle métabolique $\left(\mathrm{HbA}_{1 c} \geq 9,5 \%\right)$ en fonction du score du patient; le score de risque $\left(\mathrm{S}_{2}\right)$ est calculé à partir de la même équation : $\mathrm{S}_{2}=0,314 \times \mathrm{HbA}_{1 \mathrm{c}}+0,108 \times \mathrm{BMI}$. Reprenons le cas d'un patient (voir texte) de sexe masculin, âgé de 35 ans, diabétique depuis 10 ans (120 mois), présentant un $\mathrm{BMI}$ de $22 \mathrm{~kg} / \mathrm{m}^{2}$, un taux d'HbA $\mathrm{A}_{1 \mathrm{c}}$ de $6,9 \%$ et d'AER de $15 \mathrm{mg} / 24 \mathrm{~h}$. Le score de risque vaut: $S_{1}=S_{2}=0,314 \times 6,9+0,108 \times 22=4,5$. A 10 ans de suivi, la probabilité de développer une rétinopathie sous bon contrôle métabolique $\left(S_{1}=4,5\right.$; fig. 1a) vaut environ $21 \%$, celle d'éviter une rétinopathie sous mauvais contrôle métabolique $\left(\mathrm{S}_{2}=4,5\right.$; fig. $\left.1 \mathrm{~b}\right)$ vaut environ $33 \%$.

Figure 1: a: Probability of developing diabetic retinopathy within 5, 10 or 15 years of good metabolic control $\left(H b A_{1 c} \leq 6,9 \%\right)$ according to the patient's score; the risk score $\left(S_{1}\right)$ is calculated by the equation: $S_{1}=0,314 \times H b A_{1 c}+0,108 \times B M I$. b: Probability of not developing diabetic retinopathy within 5,10 or 15 years of poor metabolic control $\left(H b A_{1 c} \geq 9,5 \%\right)$ according to patient's score; the risk score $\left(S_{2}\right)$ is calculated by the equation: $S_{2}=0,314 \times H b A_{1 c}+0,108 \times B M I$. Consider the case of a male patient (see text), aged 35 years, with a disease duration of 120 months, a BMl of $22 \mathrm{~kg} / \mathrm{m}^{2}$, an $\mathrm{HbA}_{1 c}$ level of $6,9 \%$ and an $A E R$ of $15 \mathrm{mg} / 24 \mathrm{~h}$. The risk score equals: $\mathrm{S}_{1}=\mathrm{S}_{2}=0,314 \times 6,9+0,108 \times 22=4,5$. After 10 years of follow-up, the probability of developing retinopathy under good metabolic control $\left(S_{1}=4,5 ;\right.$ fig. 1 a) is about $21 \%$, and of not developing retinopathy under poor metabolic control $\left(S_{2}=4,5 ;\right.$ fig. $\left.1 \mathrm{~b}\right)$ is equal to $33 \%$.

Sur base des trois variables retenues, on a pu calculer le risque de développer ou de ne pas développer une rétinopathie diabétique en fonction du contrôle métabolique. À titre d'exemple, considérons un sujet de sexe masculin âgé de 35 ans, diabétique depuis l'âge de 25 ans, ayant un BMI de $22 \mathrm{~kg} / \mathrm{m}^{2}$ et suivi pendant 10 ans (tableau II). Sur base d'une valeur d'hémoglobine glycosylée défavorable au départ $\left(\mathrm{HbA}_{1 c} \geq 9,5 \%\right)$, en supposant que l'équilibre du diabète reste bon pendant les 10 ans de suivi $\left(\mathrm{HbA}_{1 c} \leq 6,9 \%\right)$, le risque de développer une rétinopathie diabétique chez ce patient atteint $38 \%$ au lieu des $10 \%$ en moyenne. À l'inverse, si l'équilibre métabolique est mauvais pendant les 10 ans de suivi $\left(\mathrm{HbA}_{1 \mathrm{c}}\right.$ $\geq 9,5 \%$ ), la probabilité d'éviter une rétinopathie diabétique est encore de $18 \%$. Supposons à présent une hé- moglobine glycosylée favorable au départ chez ce même patient $\left(\mathrm{HbA}_{1 c} \leq 6,9 \%\right)$. Dans ces conditions, si l'équilibre du diabète est mauvais pendant les 10 ans de suivi $\left(\mathrm{HbA}_{1 c} \geq 9,5 \%\right)$, il existe une chance sur trois (33\%) pour ce que patient ne développe pas une rétinopathie diabétique. À l'inverse, si l'équilibre du diabète est bon pendant les 10 ans de suivi $\left(\mathrm{HbA}_{1 c} \leq 6,9 \%\right)$, le risque de développer une rétinopathie diabétique reste élevé $(21 \%)$. Les probabilités peuvent aisément s'obtenir à partir des courbes reprises aux figures $1 a$ et $1 b$.

\section{Néphropathie [27]}

Parmi les 277 patients bien équilibrés, après 9 ans de traitement, $15 \%$ ont développé une néphropathie dia- 
Tableau I

Proportions (\%) de patients qui développent ou ne développent pas de rétinopathie ou de néphropathie diabétique en fonction du contrôle métabolique dans l'étude DCCT $[26,27]$.

Table I

Proportions (\%) of patients who developed or did not develop diabetic retinopathy or nephropathy according to metabolic control in the DCCT study.

\begin{tabular}{|c|c|c|}
\hline \multirow[b]{2}{*}{ Complication } & \multicolumn{2}{|c|}{ Contrôle métabolique } \\
\hline & $\begin{array}{c}\text { Bon } \\
\left(H b A_{1 c} \leq 6,9 \%\right)\end{array}$ & $\begin{array}{c}\text { Mauvais } \\
\left(H b A_{1 c} \geq 9,5 \%\right)\end{array}$ \\
\hline Rétinopathie (a) & $n=153$ & $\mathrm{n}=166$ \\
\hline Oui & $9,8 \%$ & $57 \%$ \\
\hline Non & $90 \%$ & $43 \%$ \\
\hline Néphropathie (b) & $\mathrm{n}=277$ & $\mathrm{n}=268$ \\
\hline Oui & $15 \%$ & $48 \%$ \\
\hline Non & $85 \%$ & $52 \%$ \\
\hline
\end{tabular}

(a) Uniquement cohorte primaire DCCT.

(b) Cohortes primaire et secondaire DCCT.

\section{Tableau II}

Probabilités (\%) de développer ou d'éviter une rétinopathie diabétique en fonction du contrôle métabolique (bon ou mauvais) chez un sujet masculin de 35 ans, diabétique depuis l'âge de 25 ans, présentant un BMI de $22 \mathrm{~kg} / \mathrm{m}^{2}$.

Table II

Probabilities (\%) of developing or avoiding diabetic retinopathy according to metabolic control (good or poor) in a 35-year old male patient, diabetic since the age of 25 , with a BMI of $22 \mathrm{~kg} / \mathrm{m}^{2}$.

\begin{tabular}{|c|c|c|c|}
\hline \multirow[b]{2}{*}{ Facteur de risque } & \multicolumn{2}{|c|}{$\begin{array}{l}\text { Contrôle } \\
\text { métabolique } \\
\text { sur } 10 \text { ans }\end{array}$} & \multirow[b]{2}{*}{$\begin{array}{l}\text { Risque } \\
\text { relatif }\end{array}$} \\
\hline & $\begin{array}{c}\text { Bon } \\
\left(H b A_{1 c}\right. \\
\leq 6,9 \%)\end{array}$ & $\begin{array}{c}\text { Mauvais } \\
\left(H b A_{1 c}\right. \\
\geq 9,5 \%)\end{array}$ & \\
\hline \multicolumn{4}{|l|}{ Favorable (a) } \\
\hline $\begin{array}{l}\text { Risque de développer } \\
\text { une rétinopathie }\end{array}$ & $21 \%$ & $67 \%$ & 3,2 \\
\hline $\begin{array}{l}\text { Risque d'éviter une rétinopathie } \\
\text { Défavorable }^{(b)}\end{array}$ & $79 \%$ & $33 \%$ & 0,42 \\
\hline $\begin{array}{l}\text { Risque de développer } \\
\text { une rétinopathie }\end{array}$ & $38 \%$ & $82 \%$ & 2,2 \\
\hline Risque d'éviter une rétinopathie & $62 \%$ & $18 \%$ & 0,29 \\
\hline
\end{tabular}

(a) Favorable : une valeur d'hémoglobine glycosylée $\left(\mathrm{HbA}_{1 c}\right)$ au départ $\leq 6,9 \%$ en plus des autres facteurs.

(b) Défavorable : une valeur d'hémoglobine glycosylée $\left(\mathrm{HbA}_{1 c}\right)$ au départ $\geq 9,5 \%$ en plus des autres facteurs. bétique malgré un bon équilibre de leur diabète. Quant aux 268 patients mal équilibrés, après 9 ans de traitement, $52 \%$ n'ont pas développé de néphropathie diabétique malgré le mauvais équilibre du diabète (tableau I). L'analyse statistique a montré qu'après avoir pris en compte le contrôle métabolique, cinq facteurs permettaient d'expliquer ces résultats surprenants, à savoir, le sexe et l'âge du patient, la présence d'une micro-albuminurie au départ de l'étude, le BMI et la durée du diabète.

Sur base de ces variables, on a pu établir le risque de développer ou de ne pas développer une néphropathie diabétique en fonction du contrôle métabolique. Considérons à nouveau à titre d'exemple le même sujet de sexe masculin âgé de 35 ans, diabétique depuis l'âge de 25 ans, ayant un BMI de $22 \mathrm{~kg} / \mathrm{m}^{2}$, et suivi pendant 10 ans (tableau III). Si, en plus des caractéristiques précitées, le patient présente une micro-albuminurie au départ élevée (AER $\geq 15 \mathrm{mg} / 24 \mathrm{~h}$ ), le risque de développer une néphropathie diabétique est de $10 \%$ lorsque le patient est bien équilibré $\left(\mathrm{HbA}_{1 c} \leq 6,9 \%\right)$ pendant les 10 ans de suivi. Avec le même profil de départ, les chances d'éviter la complication si l'équilibre du diabète est mauvais

\section{Tableau III}

Probabilités (\%) de développer ou d'éviter une néphropathie diabétique en fonction du contrôle métabolique (bon ou mauvais) chez un sujet masculin de 35 ans, diabétique depuis l'âge de 25 ans, présentant un BMI de $22 \mathrm{~kg} / \mathrm{m}^{2}$

\section{Table III}

Probabilities (\%) of developing or avoiding diabetic nephropathy according to metabolic control (good or poor) in a 35-year old male patient, diabetic since the age of 25 , with a $B M I$ of $22 \mathrm{~kg} / \mathrm{m}^{2}$.

\begin{tabular}{|c|c|c|c|}
\hline \multirow[b]{2}{*}{$\begin{array}{l}\text { Facteur } \\
\text { de risque }\end{array}$} & \multicolumn{2}{|c|}{$\begin{array}{c}\text { Contrôle } \\
\text { métabolique sur } \\
10 \text { ans }\end{array}$} & \multirow[b]{2}{*}{$\begin{array}{l}\text { Risque } \\
\text { relatif }\end{array}$} \\
\hline & $\begin{array}{c}\text { Bon } \\
\left(H b A_{1 c}\right. \\
\leq 6,9 \%)\end{array}$ & $\begin{array}{c}\text { Mauvais } \\
\left(H_{b A_{1 c}}\right. \\
\geq 9,5 \%)\end{array}$ & \\
\hline \multicolumn{4}{|l|}{ Favorable (a) } \\
\hline $\begin{array}{l}\text { Risque de développer } \\
\text { une néphropathie }\end{array}$ & $6,9 \%$ & $48 \%$ & 6,9 \\
\hline $\begin{array}{l}\text { Risque d'éviter une néphropathie } \\
\text { Défavorable }^{(b)}\end{array}$ & $93 \%$ & $52 \%$ & 0,56 \\
\hline $\begin{array}{l}\text { Risque de développer une } \\
\text { néphropathie }\end{array}$ & $10 \%$ & $61 \%$ & 6,1 \\
\hline Risque d'éviter une néphropathie & $90 \%$ & $39 \%$ & 0,43 \\
\hline
\end{tabular}

(a) Favorable : une valeur d'excrétion urinaire d'albumine (AER) au départ $\leq 7,5 \mathrm{mg} / 24 \mathrm{~h}$ outre les autres facteurs.

(b) Défavorable : une valeur d'excrétion urinaire d'albumine (AER) au départ $\geq 15 \mathrm{mg} / 24 \mathrm{~h}$ outre les autres facteurs. 

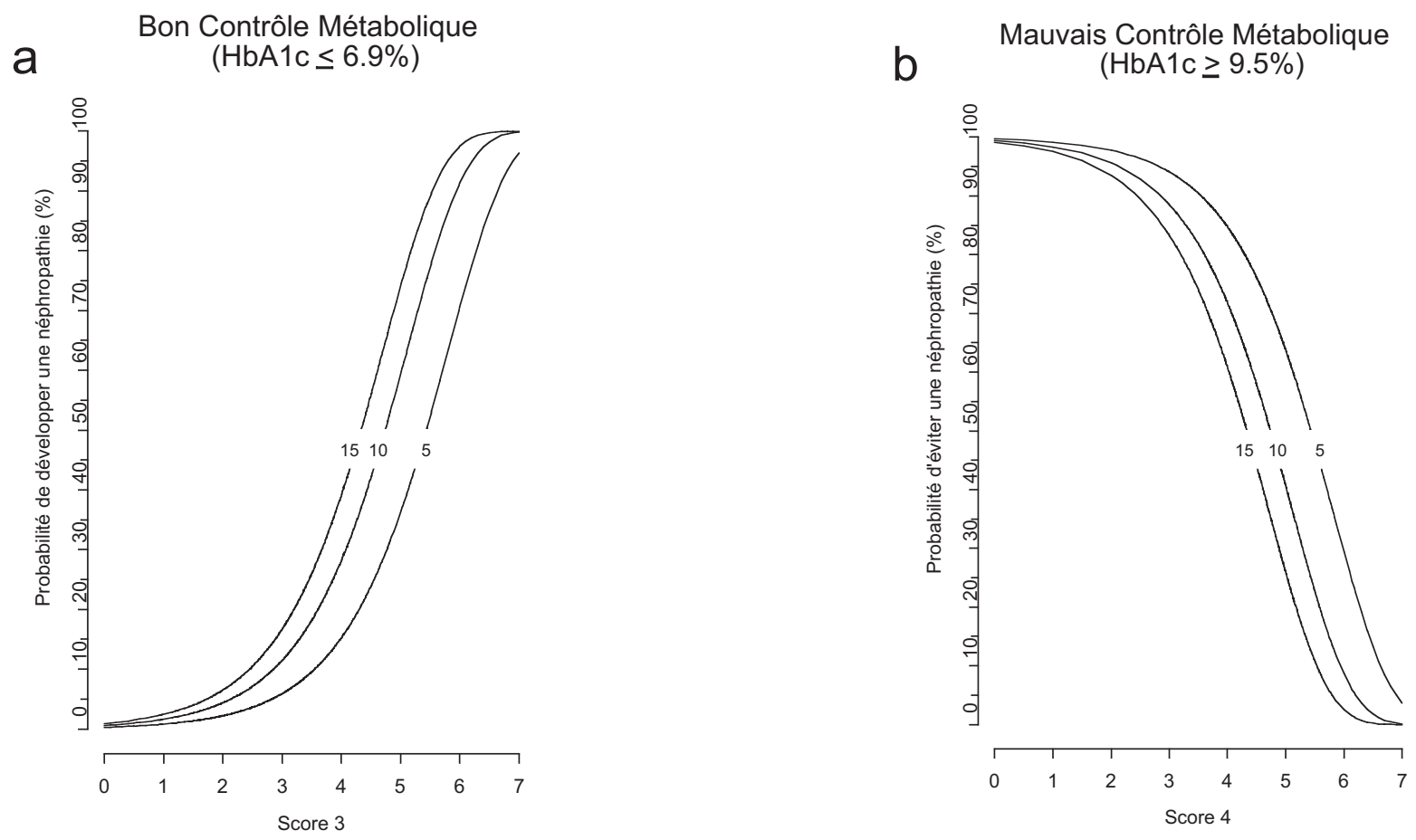

Figure 2: a : Probabilité de développer à 5, 10 ou 15 ans une néphropathie diabétique sous bon contrôle métabolique ( $\left.\mathrm{HbA}_{1 \mathrm{c}} \leq 6,9 \%\right)$ en fonction du score du patient; le score de risque $\left(S_{3}\right)$ est calculé à partir de l'équation : $S_{3}=0,54 \times \ln (A E R)+0,405 \times \ln ($ durée du diabète en mois) $-0,103 \times \mathrm{BMI}-0,0943 \times$ âge $+0,725 \times$ sexe +5 . b : Probabilité d'éviter à 5,10 ou 15 ans une néphropathie diabétique sous mauvais contrôle métabolique $\left(\mathrm{HbA}_{1 c} \geq 9,5 \%\right)$ en fonction du score du patient ; le score de risque $\left(\mathrm{S}_{4}\right)$ est calculé à partir de l'équation : $\mathrm{S}_{4}=0,54 \times$ $\ln (A E R)+0,405 \times \ln$ (durée du diabète en mois) $-0,103 \times B M I-0,0308 \times$ âge $-0,325 \times$ sexe +5 . Dans les équations $S_{3}$ et $S_{4}$, "sexe " $=0$ pour homme et 1 pour femme. Reprenons le cas d'un patient (voir texte) de sexe masculin, âgé de 35 ans, diabétique depuis 10 ans (120 mois), présentant un BMI de $22 \mathrm{~kg} / \mathrm{m}^{2}$, un taux $\mathrm{d}^{\prime} \mathrm{HbA}_{1 \mathrm{c}}$ de $6,9 \%$ et d'AER de $15 \mathrm{mg} / 24 \mathrm{~h}$. Les deux scores de risque valent respectivement : $\mathrm{S}_{3}=0,54$ $\times \ln (15)+0,405 \times \ln (120)-0,103 \times 22-0,0943 \times 35+0,725 \times 0+5=2,8 ; S_{4}=0,54 \times \ln (15)+0,405 \times \ln (120)-0,103 \times 22-0,0308 \times 35-$ $0,325 \times 0+5=5,1$. A 10 ans de suivi, la probabilité de développer une néphropathie sous bon contrôle métabolique $\left(S_{3}=2,8 ;\right.$ fig. $\left.2 a\right)$ vaut environ $10 \%$, et celle d'éviter une néphropathie sous mauvais contrôle métabolique $\left(\mathrm{S}_{4}=5,1 ;\right.$ fig. $\left.2 b\right)$ vaut environ $39 \%$.

Figure 2: a: Probability of developing diabetic nephropathy within 5, 10 or 15 years of good metabolic control ( $\left.\mathrm{HbA}_{1 \mathrm{c}} \leq 6,9 \%\right)$ according to the patient's score; the risk score $\left(S_{3}\right)$ is calculated by the equation: $S_{3}=0,54 \times \ln (A E R)+0,405 \times \ln ($ duration of diabetes in months) $-0,103 \times$ $B M I-0,0943 \times$ age $+0,725 \times$ sex +5 . $b$ : Probability of not developing diabetic nephropathy within 5,10 or 15 years of poor metabolic control $\left(H b A_{1 c} \geq 9,5 \%\right)$ according to patient's score; the risk score $\left(S_{4}\right)$ is calculated by the equation: $S_{4}=0,54 \times \ln (A E R)+0,405 \times \ln (d u r a t i o n$ of diabetes in months) $-0,103 \times B M I-0,0308 \times$ age $-0,325 \times \operatorname{sex}+5$. In the equations $S_{3}$ and $S_{4}$ "sex" = 0 for male and 1 for female. Consider the case of a male patient (see text), aged 35 years, with a disease duration of 120 months, a BMI of $22 \mathrm{~kg} / \mathrm{m}^{2}$, an $\mathrm{HbA}$, level of $6,9 \%$ and an $A E R$ of $15 \mathrm{mg} / 24 \mathrm{~h}$. The risk score equals: $S_{3}=0,54 \times \ln (15)+0,405 \times \ln (120)-0,103 \times 22-0,0943 \times 35+0,725 \times 0+5=2,8 ; S_{4}=0,54 \times \ln (15)+0,405$ $\times \ln (120)-0,103 \times 22-0,0308 \times 35-0,325 \times 0+5=5,1$. After 10 years of follow-up, the probability of developing nephropathy under good metabolic control $\left(S_{3}=2,8\right.$; fig. $\left.2 a\right)$ is about $10 \%$, and of not developing nephropathy under poor metabolic control $\left(S_{4}=5,1 ;\right.$ fig. $\left.2 b\right)$ is equal to $39 \%$.

$\left(\mathrm{HbA}_{1 \mathrm{c}} \geq 9,5 \%\right)$ pendant les 10 ans de suivi s'élèvent à $39 \%$. Si au contraire, les facteurs de risque de néphropathie diabétique au départ se présentent de façon favorable ( $A E R \leq 7,5 \mathrm{mg} / 24 \mathrm{~h}$ ) et que l'équilibre du diabète est mauvais $\left(\mathrm{HbA}_{1 \mathrm{c}} \geq 9,5 \%\right)$ pendant les 10 ans de suivi, le risque de ne pas développer une néphropathie diabétique pour ce patient est de $52 \%$ et le risque de développer la complication si l'équilibre du diabète est bon $\left(\mathrm{HbA}_{1 c} \leq 6,9 \%\right)$ pendant les 10 ans de suivi est de $7 \%$. Les probabilités peuvent aisément s'obtenir à partir des courbes reprises aux figures $2 a$ et $2 b$.

\section{CONSÉQUENCES CLINIQUES}

Le débat concernant le rôle respectif de l'équilibre métabolique du diabète et des autres facteurs de risque dans l'apparition et le développement des complications micro- et macro-angiopathiques du diabète de type 1, a toujours préoccupé les cliniciens et les chercheurs. La publication en 1993 de l'étude DCCT [18] a formellement démontré le rôle fondamental du contrôle métabolique du diabète et a montré qu'il n'y a pas de seuil glycémique, "the lowest 
the best »[20], en ce compris pour la macro-angiopathie [24]. Le suivi prolongé de la quasi totalité des patients inclus dans l'étude DCCT par l'Epidemiology of Diabetes Interventions and Complications Research Group (EDIC) [22] a permis de confirmer qu'un contrôle moins strict des deux groupes historiques du DCCT (intensif et conventionnel) conduisait à un équilibre diabétique similaire avec un taux de $\mathrm{HbA}_{1 c}$ aux environs de $8 \%$. Par ailleurs, l'effet bénéfique de 6,5 ans de bon équilibre du diabète conférait à ce groupe une protection nettement au-delà de la durée de l'étude initiale. Ceci renforce le message du rôle fondamental du bon équilibre du diabète aussi précoce et strict que possible [23]. Le rôle de la durée du diabète a également été mis en avant dans plusieurs études $[11,26,27]$. II semblerait que les premières années du diabète et plus particulièrement les années prépubertaires puissent jouer un rôle déterminant [3, $6,7]$. La place des facteurs de risque autres et indépendants de l'équilibre du diabète a également été évaluée. L'effet du tabagisme, de la pression artérielle, des lipides, du fibrinogène, du taux résiduel de C-peptide, des "Advanced Glycation Endproducts $(A G E)$ » semblerait jouer un rôle réel dans l'apparition, le développement, voire comme démontré récemment, la régression des complications diabétiques [5, 8, 9, 13-15, 25-27]. Enfin, le rôle des facteurs génétiques, qui ne sont pas actuellement modifiables, a également été précisé $[12,21]$.

Le nouveau regard que nous avons porté sur les résultats de l'étude DCCT a permis de préciser une fois encore l'importance fondamentale du bon équilibre du diabète, mais aussi l'impact d'autres facteurs de risque (BMI, taux initial de $\mathrm{HbA}_{1 c^{\prime}}$, présence ou absence de micro-albuminurie, durée d'exposition à l'hyperglycémie, âge et sexe) qui permettent d'évaluer les probabilités de développer ou de ne pas développer une rétinopathie et/ou une néphropathie diabétique. En pratique, ces probabilités s'obtiennent aisément à l'aide des nomogrammes représentés aux figures $1 a-1 b$ (rétinopathie) et aux figures $2 a-2 b$ (néphropathie). Les trois courbes sur chaque figure correspondent à des durées de suivi de 5, 10 ou 15 ans. Concrètement, on choisit la probabilité à calculer, par exemple celle d'éviter une rétinopathie diabétique chez un patient mal contrôlé pendant 5 ans (fig. 1 b), on calcule ensuite le score de risque à partir de l'équation figurant dans la légende de la figure en y insérant les caractéristiques du patient, enfin on reporte la valeur du score sur l'abscisse et on lit la probabilité sur l'ordonnée à partir de la courbe correspondante. Notons que pour la rétinopathie, l'équation utilisée est la même dans les deux cas, alors que pour le néphropathie les deux équations sont légèrement différentes car il y a interaction entre certains facteurs et le contrôle métabolique.

\section{CONCLUSIONS}

Au terme de nos études, il apparaît clairement que, tant en ce qui concerne la rétinopathie que la néphropathie diabétique, il existe, en plus du rôle déterminant de l'équilibre du diabète, d'autres facteurs indépendants liés au patient diabétique susceptibles d'influencer le devenir du patient, quant à l'apparition ou la non apparition des dites complications. L'obtention précoce et prolongée d'un contrôle métabolique optimal est donc, parallèlement à la surveillance et à la prise en compte des autres paramètres, essentielle pour la qualité de vie des patients diabétiques.

\section{RÉFÉRENCES}

1. American Diabetes Association. Clinical practice recommendations 2003. Diabetes Care 2003; 26 (suppl. 1) : S1-S156.

2. American Diabetes Association. Economic Costs of Diabetes in the U.S. in 2002. Diabetes Care $2003 ; 26: 917-932$.

3. Donaghue KC, Fairchild JM, Craig ME, et al. Do all prepubertal years of diabetes duration contribute equally to diabetes complications? Diabetes Care $2003 ; 26$ : 1224-1229.

4. European Diabetes Policy Group. A Desktop Guide to Type 1 (insulin-dependent) Diabetes Mellitus: Guidelines for Diabetes Care. Brussels, International Diabetes Federation European Region 1998.

5. Klein RL, Hunter SJ, Jenkins AJ, et al. Fibrinogen is a marker for nephropathy and peripheral vascular disease in type 1 diabetes: Studies of plasma fibrinogen and fibrinogen gene polymorphism in the DCCT/EDIC cohort. Diabetes Care 2003 ; 26 : 1439-1448.

6. Kullberg CE, Abrahamsson M, Arnqvist HJ, Finnström K, Ludvigsson J. For the Viss Study Group: Prevalence of retinopathy differs with age at onset of diabetes in a population of patients with type 1 diabetes. Diabet Med 2002; 19 : 924931.

7. Malone JI, Morrison AD, Pavan PR, Cuthbertson DD. Prevalence and significance of retinopathy in subjects with type 1 diabetes of less than 5 years' duration screened for the Diabetes Control and Complications Trial. Diabetes Care 2001 ; $24: 522-526$.

8. Orchard TJ, Forrest KY, Kuller LH, Becker DJ. How important are lipids and blood pressure for progression of complications in type 1 diabetes? International Diabetes Monitor 2002 ; 14 : 7-9.

9. Perkins BA, Ficociello LH, Silva KH, Finkelstein DM, Warram JH, Krolewski AS. Regression of microalbuminuria in type 1 diabetes. N Engl J Med 2003 ; 348 : 2285-2293.

10. Pirart J. Diabetes mellitus and its degenerative complications: a prospective study of 4,400 patients observed between 1947 and 1973. Diabetes Care 1978; 1: 168-188.

11. Porta M, Sjølie AK, Chaturvedi N, et al. Risk factors for progression to proliferative diabetic retinopathy in the EURODIAB Prospective Complications Study. Diabetologia 2001; 44: 2203-2209. 
12. Rogus JJ, Warram JH, Krolewski AS. Genetic studies of late diabetic complications: the overlooked importance of diabetes duration before complication onset. Diabetes 2002; 51 : 1655-1662.

13. Rossing $P$, Hougaard $P$, Parving $H H$. Risk factors for development of incipient and overt diabetic nephropathy in type 1 diabetic patients: a 10-year prospective observational study. Diabetes Care $2002 ; 25$ : 859-864.

14. Singh R, Barden A, Mori T, Beilin L. Advanced glycation endproducts: a review. Diabetologia $2001 ; 44$ : 129-146.

15. Steffes MW, Sibley S, Jackson M, Thomas W. $\beta$-cell function and the development of diabetes-related complications in the Diabetes Control and Complications Trial. Diabetes Care $2003 ; 26: 832-836$.

16. The DCCT Research Group. Design and methodologic considerations for the feasibility phase. Diabetes $1986 ; 35: 530$ 545.

17. The DCCT Research Group. Effects of age, duration and treatment of insulin-dependent diabetes mellitus on residual Betacell function: Observations during eligibility testing for the Diabetes Control and Complications Trial (DCCT). J Clin Endocrinol Metab $1987 ; 65: 30-36$.

18. The DCCT Research Group. The effect of intensive treatment of diabetes on the development and progression of long-term complications in insulin-dependent diabetes mellitus. N Engl J Med 1993 ; 329 : 977-986.

19. The Diabetes Control and Complications Trial Research Group. Progression of retinopathy with intensive versus conventional treatment in the Diabetes Control and Complications Trial. Ophthalmology $1995 ; 102: 647-661$.

20. The DCCT Research Group. The absence of a glycemic threshold for the development of long-term complications: the perspective of the Diabetes Control and Complications Trial. Diabetes 1996 ; 45 : 1289-1298.

21. The DCCT Research Group. Clustering of long-term complications in families with diabetes in the Diabetes Control and Complications Trial. Diabetes $1997 ; 46$ : 1829-1839.

22. The DCCT Research Group. Retinopathy and nephropathy in patients with type 1 diabetes four years after a trial of intensive therapy. N Engl J Med 2000 ; 342 : 381-389.

23. The DCCT Research Group. Effect of intensive therapy on the microvascular complications of type 1 diabetes mellitus. JAMA 2002 ; 287 : 2563-2569.

24. The DCCT Research Group. Intensive diabetes therapy and carotid intima-media thickness in type 1 diabetes mellitus. $\mathrm{N}$ Engl J Med 2003 ; 348 : 2294-2303.

25. Wautier JL, Guillausseau PJ. Advanced glycation end products, their receptors and diabetic angiopathy. Diabetes Metab $2001 ; 27: 535-542$.

26. Zhang L, Krzentowski G, Albert A, Lefèbvre PJ. Risk of developing retinopathy in Diabetes Control and Complications Trial type 1 diabetic patients with good or poor metabolic control. Diabetes Care $2000 ; 24: 1275-1279$.

27. Zhang L, Krzentowski G, Albert A, Lefèbvre PJ. Factors predictive of nephropathy in DCCT type 1 diabetic patients with good or poor metabolic control. Diabet Med $2003 ; 20: 580-$ 285 .

\section{La femme et ses hormones de 7 à 77 ans}

Principaux thèmes : Gynéco-endocrinologie de l'adolescence, Contraception (la pratique en questions-réponses), Ménopause (les THS et leurs alternatives), Ostéoporose (qui et quand dépister et comment traiter).

Vendredi 28 et samedi matin 29 janvier 2005

Espace Tête d'Or Lyon-Villeurbanne

103 Bd Stalingrad

69100 Villeurbanne

Organisation : E. Drapier-Faure

Secrétariat : Evelyne Drapier-Faure, Gynécologie,

Hôpital E. Herriot, 69437 Lyon Cedex 03 ;

tél : 0472110324 et 0472117771.

INFORMATION 